\title{
FOUR TENSORS DETERMINING THE HEAT AND ELECTRO-CONDUCTIVITIES OF DEGENERATE ELECTRONS IN THE DENSE MAGNETIZED MATTER
}

\author{
Glushikhina M. V.*广 \\ Space Research Institute of Russian Academy of Sciences, \\ Moscow, 117997, Russia \\ E-mail:m.glushikhina@iki.rssi.ru

\section{Bisnovatyi-Kogan G. S.} \\ Space Research Institute of Russian Academy of Sciences, \\ Moscow, 117997, Russia, \\ National Research Nuclear University (MEPhI), Kashirskoe Shosse, 31, \\ Moscow, 115409, Russia \\ E-mail: gkoganeiki.rssi.ru
}

\begin{abstract}
A solution is obtained for the Boltzmann equation for a plasma in a magnetic field with strongly degenerate electrons and non-degenerate nuclei. In the Lorentz approximation, the components of the diffusion, thermal diffusion, and diffusion thermoeffect tensors in a non-quantizing magnetic field are calculated. This approximation, in which electron-electron collisions are neglected, is asymptotically accurate for a plasma with highly degenerate electrons. These formulas have a much more complex dependence on the magnetic field than similar dependences in previous publications on this topic.
\end{abstract}

High Energy Phenomena in Relativistic Outflows VII - HEPRO VII

9-12 July 2019

Facultat de Física, Universitat de Barcelona, Spain

* Speaker.

${ }^{\dagger}$ A footnote may follow. 


\section{Introduction}

Heat fluxes and current densities in the crusts of neutron stars and cores of white dwarfs are determined by the coefficients of thermal conductivity, diffusion thermal effect, diffusion and thermal diffusion. To calculate them, it is necessary to know the transport properties of the dense matter of the stars, where the electrons are highly degenerate and form an almost ideal Fermi gas, and the ions are non-degenerate and form either a Coulomb liquid or a Coulomb crystal. Under these conditions, electrons, as a rule, are the most important carriers of charge and heat, and kinetic coefficients are determined mainly by the scattering of electrons by ions. Knowing the distribution of heat and current, one can calculate the magnetothermal evolution of neutron stars whose crusts form a Coulomb crystal [1]. A magnetic field limits the movement of electrons in a direction perpendicular to its lines. Since electrons are the main carriers of heat and charge, the transfer of heat and charge in this direction is suppressed, remaining unchanged along the lines. The relation between the electrical conductivity along and across the lines of the magnetic field was obtained phenomenologically in [2] in the form

$$
\frac{\sigma_{\perp}}{\sigma_{\|}}=\frac{1}{1+(\omega \tau)^{2}},
$$

and was used in [3] to calculate the kinetic coefficients of a degenerate electron gas in the crusts of neutron stars. Here $\omega=\frac{e B}{m_{e} c}$ is the cyclotron frequency of electrons, $\tau$ is the time between electron collisions, $e, m_{e}, B, c$ is the electron charge and mass, magnetic field strength and speed of light, respectively. The influence of the magnetic field on the thermal and electroconductivity in the form of (1.1) was used in many subsequent papers. In this contribution, the tensor coefficients of diffusion, thermal diffusion, and diffusion thermoeffect for highly degenerate electrons in the Lorentz approximation are presented. For the first time, asymptotically accurate analytical expressions for these kinetic coefficients in the presence of a magnetic field are obtained. These expressions are much more complicated depending on the magnetic field than in previous works on this topic. The use of kinetic coefficients calculated in this article will make it possible to more accurately take into account the processes occurring in the crust of a neutron star. The obtained expressions can be used to describe the transfer coefficients in other magnetized objects containing free degenerate electrons.

\section{Boltzmann equation and conditions of applicability}

The Boltzmann equation, that describes the change in the distribution function of electrons $f$ over time, in the presence of electric and magnetic fields, is written as [4], [5]

$$
\frac{\partial f}{\partial t}+c_{i} \frac{\partial f}{\partial r_{i}}-\left[\frac{e}{m_{e}}\left(E_{i}+\frac{1}{c} \varepsilon_{i k l} c_{k} B_{l}\right)\right] \frac{\partial f}{\partial c_{i}}+J=0 .
$$

Here $(-e), m_{e}$ is the charge (negative) and mass of the electron, $E_{i}, B_{i} \quad-$ electric field strength and magnetic induction, $\varepsilon_{i k l}$ completely antisymmetric Levi-Civita tensor, $c-$ speed of light. The electron distribution is determined by the Fermi-Dirac function. 
The collision integral $J$ for arbitrary electron degeneracy, according to $[6,7,8,9]$, is written in the form:

$$
\begin{array}{r}
J=J_{e e}+J_{e N}=R \int\left[f^{\prime} f_{1}^{\prime}(1-f)\right. \\
\left.+\left(1-f_{1}\right)-f f_{1}\left(1-f^{\prime}\right)\left(1-f_{1}^{\prime}\right)\right] \times g_{e e} b d b d \varepsilon d c_{1 i}+ \\
+\int\left[f^{\prime} f_{N}^{\prime}(1-f)-f f_{N}\left(1-f^{\prime}\right)\right] \times g_{e N} b d b d \varepsilon d c_{N i} .
\end{array}
$$

Here, the impact parameter $b$, and $\varepsilon$ are geometrical parameters of particle collisions with relative velocities $g_{e e}, g_{e N}, R=\frac{2 m_{e}^{3}}{h^{3}}$. The integration in electron part of the collision integral in (2.2) is performed over the phase space of the incoming particles $\left(d c_{1 i}\right)$, and the physical space of their arrival $(b d b d \varepsilon)$ [8].

The Boltzmann equation for electrons with the pair collision integral (2.2) can be used when electronic gas is considered almost perfect, i.e. the kinetic energy of electrons is much greater than energy of electrostatic interactions. This is true for a plasma of sufficiently low density. On the contrary in neutron stars and white dwarfs: the plasma has a very high density, at which it is important to take into account the degeneracy of electrons. It is known from statistical physics that a gas of highly degenerate electrons becomes ideal, since large Fermi energies in this case replace thermal energy [10]. Therefore, the calculations in this paper are valid for a low-density plasma, as well as for a plasma with a high density and degenerate electrons. A detailed discussion of the applicability of the pair collision integral (2.2) and its modifications for non-degenerate highdensity gases can be found in [8].

The collision integral similar to $J_{e e}$ from (2.2) for strongly degenerate neutrons in nuclear matter is given in [9], see also [11]. In the presence of non-degenerate heavy nuclei and highly degenerate neutrons, the contribution of collisions between them to the heat transfer and diffusion coefficients is negligible compared to neutron-nucleus collisions. The same situation holds for highly degenerate electrons. Therefore, for strongly degenerate electrons, the Lorentz approximation, taking into account collisions between light and heavy particles, is asymptotically accurate. Therefore, for our consideration, we can neglect $J_{e e}$ compared to $J_{e N}$, and we can equate $J=J_{e N}$ in (2.2).

The transfer equations for the electron concentration, total momentum, and electron energy, in the two-component mixture of electrons and nuclei, can be obtained in a usual manner from the Boltzmann equation in a quasi-neutral plasma $[8,4,5]$.

\section{Solution method}

The Boltzmann equation can be solved by using the Chapman-Enskog method of successive approximations [8]. This method is used when the distribution functions are close to their values in the state of thermodynamic equilibrium, and deviations are considered in a linear approximation. In a first approximation, we look for the distribution function $f$ in the form $f=f_{0}\left[1+\chi\left(1-f_{0}\right)\right]$

The $\chi$ function allows the solution to be represented as [13]:

$$
\chi=-A_{i} \frac{\partial \ln T}{\partial r_{i}}-n_{e} D_{i} d_{i} \frac{G_{5 / 2}}{G_{3 / 2}}
$$

where diffusion vector $d_{i}$ : 


$$
d_{i}=\frac{\rho_{N}}{\rho} \frac{\partial \ln P_{e}}{\partial r_{i}}-\frac{\rho_{e}}{P_{e}} \frac{1}{\rho} \frac{\partial P_{N}}{\partial r_{i}}+\frac{e n_{e}}{P_{e}}\left(E_{i}+\frac{1}{c} \varepsilon_{i k l} c_{0 k} B_{l}\right) .
$$

The functions $A_{i}$ and $D_{i}$ determine the heat flux and diffusion. At $[4,5]$ it is shown that in the presence of a magnetic field with an axial vector $B_{i}$, the polar vectors $A_{i} D_{i}$ can be searched for in the form:

$$
A_{i}=A^{(1)} v_{i}+A^{(2)} \varepsilon_{i j k} v_{j} B_{k}+A^{(3)} B_{i}\left(v_{j} B_{j}\right), \quad D_{i}=D^{(1)} v_{i}+D^{(2)} \varepsilon_{i j k} v_{j} B_{k}+D^{(3)} B_{i}\left(v_{j} B_{j}\right),
$$

Introducing functions $\xi_{A}=A^{(1)}+i B A^{(2)}, \xi_{D}=D^{(1)}+i B D^{(2)}$ and dimensionless velocity: $u_{i}=$ $\sqrt{\frac{m_{e}}{2 k T}} v_{i}$, we will get, omitting small terms on the order of $\sim \frac{m_{e}}{m_{N}}$, the equations for $\xi_{A}$ and $\xi_{D}$ :

$$
\begin{gathered}
f_{0}\left(1-f_{0}\right)\left(u^{2}-\frac{5 G_{5 / 2}}{2 G_{3 / 2}}\right) u_{i}=-i B f_{0}\left(1-f_{0}\right) \frac{e \xi_{A}}{m_{e} c} u_{i}+I_{e N}\left(\xi_{A} u_{N i}\right), \\
f_{0}\left(1-f_{0}\right) u_{i}=-i B f_{0}\left(1-f_{0}\right) \frac{e \xi_{D}}{m_{e} c} u_{i}+I_{e N}\left(\xi_{D} u_{N i}\right),
\end{gathered}
$$

where

$$
I_{e N}\left(\xi u_{N i}\right)=\int f_{0} f_{N 0}\left(1-f_{0}^{\prime}\right)\left(\xi u_{i}-\xi^{\prime} u_{i}^{\prime}\right) g_{e N} b d b d \varepsilon d c_{N i}
$$

\section{Coefficients of thermal diffusion, diffusion, and diffusion thermoeffect of degenerate electrons in a magnetic field}

The general relations for the heat flux $q_{i}$ and the average directional (diffusion) electron velocity $\left\langle v_{i}\right\rangle$ are written as

$$
\begin{gathered}
q_{i}=-\lambda_{i j} \frac{\partial T}{\partial x_{j}}-n_{e} \frac{G_{5 / 2}}{G_{3 / 2}} v_{i j} d_{j}=q_{i}^{(A)}+q_{i}^{(D)}, \\
\left\langle v_{i}\right\rangle=-\mu_{i j} \frac{\partial T}{\partial x_{j}}-n_{e} \frac{G_{5 / 2}}{G_{3 / 2}} \eta_{i j} d_{j}=\left\langle v_{i}^{(A)}\right\rangle+\left\langle v_{i}^{(D)}\right\rangle,
\end{gathered}
$$

where $\lambda_{i j}$ and $v_{i j}$ are the tensors of thermal conductivity and diffusion thermal effect, and $\mu_{i j}$ and $\eta_{i j}$ are the thermal diffusion and diffusion tensors, respectively [11], [12]. The indices $(A)$ and $(D)$ determine the heat fluxes and diffusion velocities of electrons, determined by the temperature gradient $\frac{\partial T}{\partial x_{j}}$, and the diffusion vector $d_{j}$, respectively.

The method for calculating the coefficients of the thermal conductivity tensor $\lambda_{i j}$ is described in detail in [13], where analytical expressions are obtained for them. Similarly, detailed description of calculating the coefficients of the tensors $\mu_{i j}, v_{i j}$ and $\eta_{i j}$, can be found in [14].

The frequency of electron-ion collisions $v_{e i}$ was expressed in [15] and in the limiting case for degenerate matter can be written as

$$
v_{e i}=\frac{32 \pi^{2}}{3} m_{e} \frac{Z^{2} \mathrm{e}^{4} \Lambda n_{N}}{h^{3} n_{\mathrm{e}}} \quad(D) \quad \tau_{d}=1 / v_{d}
$$

The obtained results of calculations, for the components of thermal diffusion tensor: 


$$
\begin{gathered}
\mu^{(1)}=\frac{4 \pi^{3}}{3} \frac{k^{2} T}{n_{e} h^{2}}\left(\frac{3 n_{e}}{\pi}\right)^{1 / 3} \tau_{d}\left[\frac{1}{1+\omega^{2} \tau_{d}^{2}}-\frac{2 \omega^{2} \tau_{d}^{2}}{\left(1+\omega^{2} \tau_{d}^{2}\right)^{2}}-\left.\frac{\pi^{2}}{6}\left(\frac{1}{1+\omega^{2} \tau_{d}^{2} \frac{y^{3}}{x_{0}^{3}}}\right)\right|_{y=x_{0}}\right], \\
\mu^{(2)}=-\frac{4 \pi^{3}}{3} \frac{k^{2} T}{n_{e} h^{2}}\left(\frac{3 n_{e}}{\pi}\right)^{1 / 3} \frac{\omega \tau_{d}^{2}}{B}\left[\frac{2}{1+\omega^{2} \tau_{d}^{2}}-\frac{2 \omega^{2} \tau_{d}^{2}}{\left(1+\omega^{2} \tau_{d}^{2}\right)^{2}}-\left.\frac{\pi^{2}}{6}\left(\frac{1}{1+\omega^{2} \tau_{d}^{2} \frac{y^{3}}{x_{0}^{3}}}\right)\right|_{y=x_{0}} ^{\prime \prime}\right] \\
B^{2} \mu^{(3)}=\mu^{(1)}(B=0)-\mu^{(1)} .
\end{gathered}
$$

Dependence of $\mu_{\perp} / \mu_{\|}$from $\omega \tau_{d}$ for comparison with the results of previous studies on this matter is plotted on the Fig.1 on the left.

Components of the diffusion tensor:

$$
\begin{gathered}
\eta^{(1)}=\frac{k T}{n_{e} m_{e}} \tau_{d}\left(\frac{1}{1+\omega^{2} \tau_{d}^{2}}+\left.\frac{\pi^{2}}{6}\left(\frac{1}{1+\omega^{2} \tau_{d}^{2}\left(y^{3} / x_{0}^{3}\right)}\right) "\right|_{x=x_{0}}\right), \\
\eta^{(2)}=-\frac{k T}{n_{e} m_{e}} \frac{\omega \tau_{d}^{2}}{B}\left(\frac{1}{1+\omega^{2} \tau_{d}^{2}}+\left.\frac{\pi^{2}}{6}\left(\frac{1}{1+\omega^{2} \tau_{d}^{2}\left(y^{3} / x_{0}^{3}\right)}\right) "\right|_{x=x_{0}}\right), \\
B^{2} \eta^{(3)}=\eta^{(1)}(B=0)-\eta^{(1)} .
\end{gathered}
$$

Components of the diffusion thermal effect tensor:

$$
\begin{gathered}
v^{(1)}=\frac{k T h^{2}}{8 m_{e}^{2}}\left(\frac{3 n_{e}}{\pi}\right)^{2 / 3} \tau_{d}\left(\frac{1}{1+\omega^{2} \tau_{d}^{2}}+\left.\frac{\pi^{2}}{6}\left(\frac{1}{1+\omega^{2} \tau_{d}^{2}\left(y^{3} / x_{0}^{3}\right)}\right) ”\right|_{x=x_{0}}\right), \\
v^{(2)}=-\frac{k T h^{2}}{8 m_{e}^{2}}\left(\frac{3 n_{e}}{\pi}\right)^{2 / 3} \frac{\omega \tau_{d}^{2}}{B}\left(\frac{1}{1+\omega^{2} \tau_{d}^{2}}+\left.\frac{\pi^{2}}{6}\left(\frac{1}{1+\omega^{2} \tau_{d}^{2}\left(y^{3} / x_{0}^{3}\right)}\right) "\right|_{x=x_{0}}\right), \\
B^{2} v^{(3)}=v^{(1)}(B=0)-v^{(1)} .
\end{gathered}
$$

Here $x_{0}=\mu / k T$ is rate of degeneracy, $\mu$ is the chemical potential of electrons, and $y=u^{2}$ is squared dimentionless velocity.

The dependences of $v_{\perp} / v_{\|}$and $\eta_{\perp} / \eta_{\|}$from $\omega \tau_{d}$ are plotted on the Fig. 1 and 2.

If the exact solution (for diffusion and diffusional thermal effect) takes into account terms of the order of $1 / x_{0}^{2}$ small, then the solution obtained from the Boltzmann equation will differ from the phenomenological (1.1).

For completeness, we give the expressions for the coefficients of the tensor $\lambda_{i j}$ in a strongly degenerate plasma from [13] 


$$
\begin{gathered}
\lambda^{(1)}=\frac{5 \pi^{2}}{6} \frac{k^{2} T n_{e}}{m_{e}} \tau_{d}\left\{\frac{1}{1+\omega^{2} \tau_{d}^{2}}-\frac{6}{5} \frac{\omega^{2} \tau_{d}^{2}}{\left(1+\omega^{2} \tau_{d}^{2}\right)^{2}}-\left.\frac{\pi^{2}}{10}\left[\frac{1}{1+\omega^{2} \tau_{d}^{2}\left(\frac{y^{3}}{x_{0}^{3}}\right)}\right]^{\prime \prime}\right|_{x=x_{0}}\right\}, \\
\lambda^{(2)}=-\frac{4 \pi^{2}}{3} \frac{k^{2} T n_{e}}{m_{e}} \frac{\tau_{d}^{2}}{B}\left\{\frac{1}{1+\omega^{2} \tau_{d}^{2}}-\frac{3}{4} \frac{\omega^{2} \tau_{d}^{2}}{\left(1+\omega^{2} \tau_{d}^{2}\right)^{2}}-\left.\frac{\pi^{2}}{16}\left[\frac{1}{1+\omega^{2} \tau_{d}^{2}\left(\frac{y^{3}}{x_{0}^{3}}\right)}\right]^{\prime \prime}\right|_{x=x_{0}}\right\}, \\
B^{2} \lambda^{(3)}=\lambda^{(1)}(B=0)-\lambda^{(1)} .
\end{gathered}
$$
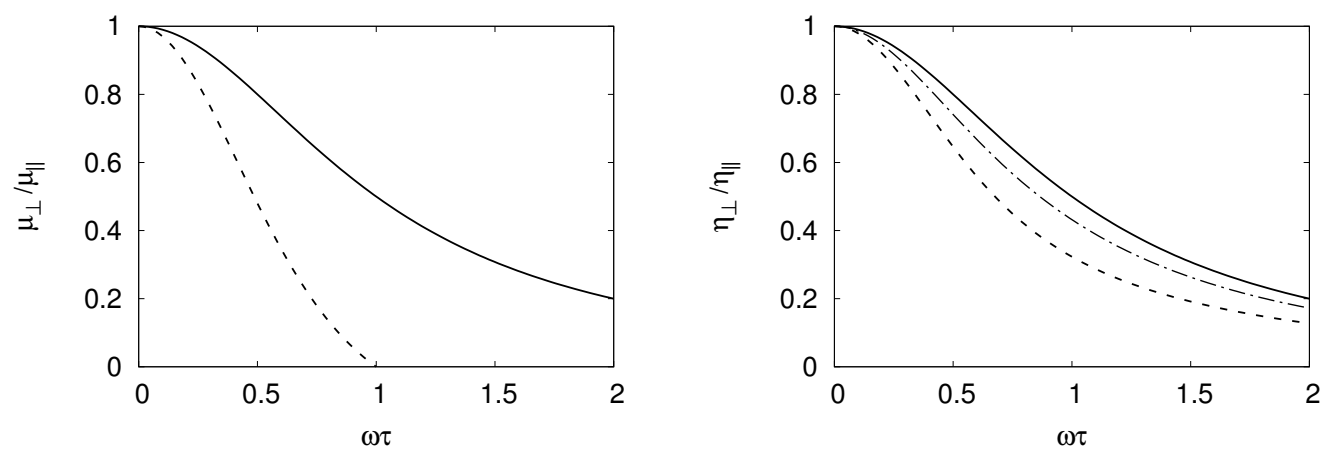

Figure 1: The left figure is the ratio $\mu_{\perp} / \mu_{\|}$as a function of $\omega \tau$. For comparison, the curves representing the phenomenologically determined thermal diffusion coefficient (solid curve) and that obtained from the asymptotic solution to the Boltzmann equation (dashed curve) are shown. The right figure is the ratio $\eta_{\perp} / \eta_{\|}$ as a function of $\omega \tau$. For diffusion, the phenomenologically obtained solid curve coincides with the curve obtained by solving the Boltzmann equation in the case of strong degeneracy. If terms on the order of smanless of $1 / x_{0}^{2}$ are retained in the exact solution, then the solution obtained from the Boltzmann equation differs from the phenomenological one given by (1.1). The dash-dotted and dashed curves correspond to the solutions obtained with the allowance for small terms at $k T=0.11 E_{f} \quad\left(x_{0}=9\right)$ and $k T=0.2 E_{f} \quad\left(x_{0}=5\right)$ respectively.

\section{Conclusion}

In our work, we find the tensors of kinetic coefficients of diffusion, thermal diffusion, and diffusion thermoeffect for highly degenerate nonrelativistic electrons in a non-quantizing magnetic field. The solution was obtained asymptotically precisely in the Lorentz approximation, when electron-electron collisions can be neglected in comparison with electron-nucleus collisions. The tensors are obtained for arbitrary directions of the magnetic field and temperature gradient in the Cartesian coordinate system, according to [4]. In most works dealing with kinetic coefficients in astrophysical objects, in particular, thermal conductivity and electrical conductivity in neutron stars, the influence of the magnetic field was taken into account phenomenologically using the coefficient $1 /\left(1+\omega^{2} \tau^{2}\right)$, which reduces heat flux and diffusion in the direction perpendicular to the 

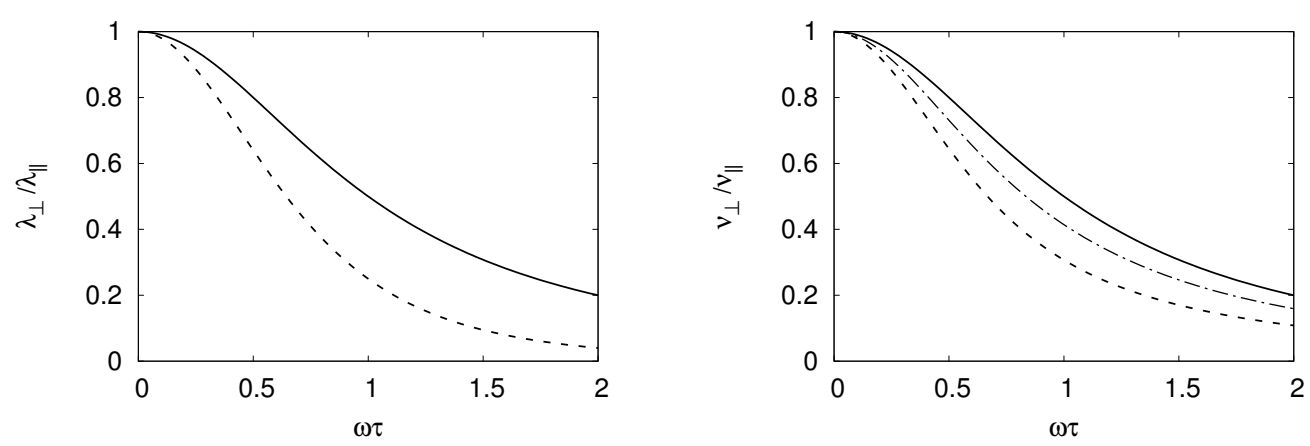

Figure 2: The left figure is ratio $\lambda_{\perp} / \lambda_{\|}$as a function of $\omega \tau$. For comparison, the curves representing the phenomenologically determined thermal conductivity (solid curve) and the asymptotic thermal conductivity obtained by solving the Boltzmann equation (dashed curve) are shown. The right figure is ratio $v_{\perp} / v_{\|}$as a function of $\omega \tau$. Similar to diffusion, the phenomenologically obtained solid curve coinsides with the asymptotic solution for the diffusion thermoeffect. If terms on the order of smalness of $1 / x_{0}^{2}$ are retained in the solution obtained from the Boltzmann equation, then the plot of $v_{\perp} / v_{\|}$differs from the phenomenological curve. The dash-dotted and dashed curves correspond to the solutions obtained at $k T=0.11 E_{f} \quad\left(x_{0}=9\right)$ and $k T=0.2 E_{f} \quad\left(x_{0}=5\right)$, respectively.

direction of the magnetic field [2], [3]. Our results obtained by solving the Boltzmann equation show that the influence of the magnetic field on the kinetic coefficients is stronger and has a more complex character. Calculations were made for nonrelativistic electrons, although relativistic effects become important in the deep layers of the neutron star's crust.

Electrical conductivity of relativistic degenerate electron gas in intense magnetic field was considered by Canuto [16, 17].

The main relativistic effect of an increase in the effective electron mass can be approximately taken into account by replacing, in all expressions, the rest mass of the electron $m_{e}$ with the relativistic mass of the electron $m_{e *}=\left(m_{e}^{2}+p_{F e}^{2} / c^{2}\right)^{1 / 2} \quad$ [3]. The diffusion vector, which determines the electric current in a medium with gradients of various parameters and a nonzero electric field, is important for calculating the geometry and evolution of the magnetic field in degenerate stars. The obtained kinetic coefficients can be used to calculate the fluxes of heat and electric current in white dwarfs, on the surface and in the crust of neutron stars, as well as in a magnetized plasma incident on a neutron star.

\section{Acknowledgments}

This work was supported by a grant from the Russian Foundation for Basic Research. No. 17-02-00760 and by the program of the Russian Academy of Sciences P-12.

\section{References}

[1] E.E. Salpeter, Astrophysical Journal 134, 669 (1961).

[2] E. Flowers, N. Itoh, Astrophysical Journal 206, 218 (1976).

[3] D.G. Yakovlev, V.A. Urpin, Astron. Zh. 57, 526 (1980). 
[4] G. S. Bisnovatyi-Kogan, Transport properties of partially ionized two-temperature plasma and isotropic corrections to maxvellian distribution function. (Diploma thesis, Moscow Institute of Physics and Technology, 1964).

[5] G. S. Bisnovatyi-Kogan, J. Appl. Mech. Tech. Phys., 3, 43 (1964).

[6] E. Uehling, G. Uhlenbeck, Phys. Rev. 43, 552 (1933).

[7] E. Uehling, Phys. Rev. 46, 917 (1934).

[8] S. Chapmen, T. G. Cowling, Mathematical Theory of Nonuniform Gases, Cambrige, 1952 [Russian translation published by IL, Moscow, 1958].

[9] S.Z. Tomonaga, Z. Phys. 110, 573 (1938).

[10] L. D. Landau, E.M. Lifshitz, Statistical Physics, 5, 3rd ed., Butterworth-Heinemann, 1980.

[11] G. S. Bisnovatyi-Kogan, M.M. Romanova, JETP 56, 243 (1983).

[12] L.E. Kalikhman, Elements of magnetohydrodynamics, Atomizdat, Moscow, 1964.

[13] G. S. Bisnovatyi-Kogan, M.V. Glushikhina, Plasma Physics Reports 44, 405 (2018);

[14] G. S. Bisnovatyi-Kogan, M.V. Glushikhina, Plasma Physics Reports 44, 1114 (2018);

[15] V.A. Ginzburg, A.A. Rukhadze, Waves in Magnetoactive Plasma, Nauka, Moscow 1970.

[16] V. Canuto, C. Chiuderi, Phys. Rev. 1, 2219 (1970).

[17] V. Canuto, Astrophysical Journal 159, 641 (1970). 